(C) 1998 International Press

Adv. Theor. Math. Phys. 2 (1998) 219-229

\title{
Quantum Cohomology at Higher Genus: Topological Recursion Relations and Virasoro Conditions
}

\author{
Tohru Eguchi ${ }^{a}$, Chuan-Sheng Xiong $^{b}$ \\ ${ }^{a}$ Department of Physics, Faculty of Science, \\ University of Tokyo, \\ Tokyo 113, Japan \\ ${ }^{b}$ Yukawa Institute for Theoretical Physics, \\ Kyoto University \\ Kyoto 606, Japan
}

\begin{abstract}
We construct topological recursion relations (TRR's) at higher genera $g \geq 2$ for general 2-dimensional topological field theories coupled to gravity. These TRR's when combined with Virasoro conditions enable one to determine the number of higher genus holomorphic curves in any Fano varieties. In the case of $C P^{2}$ we reproduce the known results at genus $g=2$.
\end{abstract}

\section{Introduction}

Recently we have proposed that the partition functions of topological string theories compactified on an arbitrary Kähler manifold are highest-weight states of a Virasoro algebra [1-3]. We have shown that the Virasoro conditions together with topological recursion relations reproduce known instanton numbers (numbers of holomorphic curves) at genus 0 and 1 of various Fano varieties. Topological recursion relations (TRR's) exist at genus $g=0$ and $g=1$ [4] and convert correlation functions of gravitational descendants into those of primary fields which may be evaluated directly by the method 
of algebraic geometry. In the case of higher-genera $g \geq 2$, however, no TRR have been so far available and it was not possible to compare the predictions of Virasoro conditions with the known geometrical data on the higher genus curves.

Recently Getzler has announced the existence of a TRR at genus $g=2$ [5] which involves descendants of degree $n, n-1$ and $n-2$. His analysis is based on the study of a linear relation among homology cycles on the moduli space of holomorphic maps. A precise form of his formula, however, is not yet available.

In this paper we propose a somewhat different form of TRR at higher genera starting from a simple assumption on the dependence of higher-genus free energies on genus $=0$ primary correlation functions. Our recursion relation at genus $g$ involves gravitational descendants of degree $n, n-1, \cdots, n-3 g+1$. In the case of genus 2 descendants of degree $n, n-1, \cdots, n-5$ appear and thus our relation is somewhat weaker than that of Getzler's. Nevertheless, these TRR's can be used together with the Virasoro conditions in order to completely determine the number of holomorphic curves of arbitrary degree and genus. In the case of $C P^{2}$ at genus $g=2$ we explicitly verify that our TRR and Virasoro conditions reproduce the known results of [6-8].

\section{Topological Recursion Relation at Higher Genus}

Our basic assumption is that the genus- $g$ free energy of topological string theory is a function depending only on the primary multi-point functions of genus $g=0$. Let us consider the case of a theory with primary fields $\left\{\mathcal{O}_{\alpha}\right\}(\alpha=0,1 \cdots, N)$ coupled to the perturbation parameters $\left\{t^{\alpha}\right\}$. Gravitational descendants and their couplings are denoted as $\left\{\sigma_{n}\left(\mathcal{O}_{\alpha}\right)\right\}$ and $\left\{t_{n}^{\alpha}\right\}$ $(n=0,1,2, \cdots)$, respectively. We define genus $=0$ correlation functions as $u_{\alpha_{1} \alpha_{2} \cdots \alpha_{j}} \equiv\left\langle P \mathcal{O}_{\alpha_{1}} \mathcal{O}_{\alpha_{2}} \cdots \mathcal{O}_{\alpha_{j}}\right\rangle_{0}$ where $P$ denotes the puncture operator. Our assumption is that the genus- $g$ free energy $F_{g}$ is a function only of the genus $=0$ correlation functions $u_{\alpha_{1}}, u_{\alpha_{1} \alpha_{2}}, \cdots, u_{\alpha_{1} \alpha_{2} \cdots \alpha_{3 g-1}}$

$$
F_{g}(t)=F_{g}\left(u_{\alpha_{1}}(t), u_{\alpha_{1} \alpha_{2}}(t), \cdots, u_{\alpha_{1} \alpha_{2} \cdots \alpha_{3 g-1}}(t)\right), \quad g \geq 1 .
$$

Here $t$ stands for all the couplings $\left\{t_{n}^{\alpha}\right\}$ of the large phase space. Note that the dependence on the parameters $t$ of the free energy $F_{g}(t)$ occurs only through the functions $u_{\alpha_{1} \alpha_{2} \cdots \alpha_{j}}(t)(j=1,2, \cdots, 3 g-1)$. 
Equation (2.1) is known to hold in the 2-dimensional pure gravity theory. For instance, the genus 1,2 and 3 free energies are given by $[9,10]$

$$
\begin{aligned}
F_{1}= & \frac{1}{24} \log u^{\prime}, \quad F_{2}=\frac{u^{\prime \prime 3}}{360 u^{\prime 4}}-\frac{7 u^{\prime \prime} u^{(3)}}{1920 u^{\prime 3}}+\frac{u^{(4)}}{1152 u^{\prime 2}} \\
F_{3}= & -\frac{5 u^{\prime \prime}}{648 u^{\prime 8}}+\frac{59 u^{\prime \prime} u^{(3)}}{3024 u^{\prime 7}}-\frac{83 u^{\prime 2} u^{(3)^{2}}}{7168{u^{\prime}}^{6}}+\frac{59 u^{(3)^{3}}}{64512 u^{\prime 5}}-\frac{83 u^{\prime \prime 3} u^{(4)}}{15120 u^{\prime 6}} \\
& +\frac{1273 u^{\prime \prime} u^{(3)} u^{(4)}}{322560 u^{\prime 5}}-\frac{103 u^{(4)^{2}}}{483840 u^{\prime 4}}+\frac{353 u^{\prime \prime} u^{(5)}}{322560 u^{\prime 5}}-\frac{53 u^{(3)} u^{(5)}}{161280 u^{\prime 4}}-\frac{7 u^{\prime \prime} u^{(6)}}{46080 u^{\prime 4}} \\
& +\frac{u^{(7)}}{82944 u^{\prime 3}}
\end{aligned}
$$

where $u=\langle P P\rangle_{0}$ and ' denotes the $t^{0}$-derivative. Eq. (2.1) is also known to hold in some cases of the 2-dimensional gravity coupled to minimal matter at lower genera [11]. It is also valid in the case of $C P^{1}$ model [12]. Eq. (2.1) means that higher genus amplitudes are expressed in terms of the genus $=0$ data and suggests a possible reinterpretation of the world-sheet topological theory as a field theory on the target space $[10,11]$. We now assume that eq. (2.1) is a universal feature of 2-dimensional topological field theories coupled to gravity.

It is then easy to derive our TRR's. Let us first consider for simplicity the case of genus $=1$. Genus- 1 free energy depends on $\left\{u_{\alpha}\right\}$ and $\left\{u_{\alpha \beta}\right\}$. We then have

$$
\begin{aligned}
\frac{\partial F_{1}}{\partial t_{n}^{\alpha}}=\frac{\partial u_{\mu}}{\partial t_{n}^{\alpha}} \frac{\partial F_{1}}{\partial u_{\mu}}+\frac{\partial u_{\mu \nu}}{\partial t_{n}^{\alpha}} \frac{\partial F_{1}}{\partial u_{\mu \nu}}=\left\langle\sigma_{n}\left(\mathcal{O}_{\alpha}\right) \mathcal{O}_{\mu} P\right\rangle_{0} \frac{\partial F_{1}}{\partial u_{\mu}} & \\
& +\frac{\partial\left\langle\sigma_{n}\left(\mathcal{O}_{\alpha}\right) \mathcal{O}_{\nu} P\right\rangle_{0}}{\partial t_{\mu}} \frac{\partial F_{1}}{\partial u_{\mu \nu}}
\end{aligned}
$$

At $n=0$ eq.(2.4) becomes

$$
\left\langle\mathcal{O}_{\alpha}\right\rangle_{1}=\left\langle\mathcal{O}^{\alpha} \mathcal{O}_{\mu} P\right\rangle_{0} \frac{\partial F_{1}}{\partial u_{\mu}}+\left\langle\mathcal{O}^{\alpha} \mathcal{O}_{\mu} \mathcal{O}_{\nu} P\right\rangle_{0} \frac{\partial F_{1}}{\partial u_{\mu \nu}} .
$$

We use the genus $=0 \mathrm{TRR}$

$$
\left\langle\sigma_{n}\left(\mathcal{O}_{\alpha}\right) A B\right\rangle_{0}=\left\langle\sigma_{n-1}\left(\mathcal{O}_{\alpha}\right) \mathcal{O}_{\gamma}\right\rangle_{0}\left\langle\mathcal{O}^{\gamma} A B\right\rangle_{0}
$$

to rewrite $(2.4)$ as

$$
\begin{aligned}
& \left\langle\sigma_{n}\left(\mathcal{O}_{\alpha}\right)\right\rangle_{1}=\left\langle\sigma_{n-1}\left(\mathcal{O}_{\alpha}\right) \mathcal{O}_{\beta}\right\rangle_{0}\left\langle\mathcal{O}^{\beta} \mathcal{O}_{\mu} P\right\rangle_{0} \frac{\partial F_{1}}{\partial u_{\mu}} \\
+ & \left(\left\langle\sigma_{n-1}\left(\mathcal{O}_{\alpha}\right) \mathcal{O}_{\beta}\right\rangle_{0}\left\langle\mathcal{O}^{\beta} \mathcal{O}_{\mu} \mathcal{O}_{\nu} P\right\rangle_{0}+\left\langle\sigma_{n-1}\left(\mathcal{O}_{\alpha}\right) \mathcal{O}_{\mu} \mathcal{O}_{\beta}\right\rangle_{0}\left\langle\mathcal{O}^{\beta} \mathcal{O}_{\nu} P\right\rangle_{0}\right) \frac{\partial F_{1}}{\partial u_{\mu \nu}}
\end{aligned}
$$


Putting $n=1$ in (2.7) gives

$$
\begin{aligned}
& \left\langle\sigma_{1}\left(\mathcal{O}_{\alpha}\right)\right\rangle_{1}=\left\langle\mathcal{O}_{\alpha} \mathcal{O}_{\beta}\right\rangle_{0}\left\langle\mathcal{O}^{\beta} \mathcal{O}_{\mu} P\right\rangle_{0} \frac{\partial F_{1}}{\partial u_{\mu}} \\
& +\left(\left\langle\mathcal{O}_{\alpha} \mathcal{O}_{\beta}\right\rangle_{0}\left\langle\mathcal{O}^{\beta} \mathcal{O}_{\mu} \mathcal{O}_{\nu} P\right\rangle_{0}+\left\langle\mathcal{O}_{\alpha} \mathcal{O}_{\mu} \mathcal{O}_{\beta}\right\rangle_{0}\left\langle\mathcal{O}^{\beta} \mathcal{O}_{\nu} P\right\rangle_{0}\right) \frac{\partial F_{1}}{\partial u_{\mu \nu}} \\
& =\left\langle\mathcal{O}_{\alpha} \mathcal{O}_{\beta}\right\rangle_{0}\left\langle\mathcal{O}^{\beta}\right\rangle_{1}+\left\langle\mathcal{O}_{\alpha} \mathcal{O}_{\mu} \mathcal{O}_{\beta}\right\rangle_{0}\left\langle\mathcal{O}^{\beta} \mathcal{O}_{\nu} P\right\rangle_{0} \frac{\partial F_{1}}{\partial u_{\mu \nu}}
\end{aligned}
$$

where (2.5) has been used. By making use of (2.5) and (2.8) eq.(2.7) is then reexpressed as

$$
\begin{aligned}
\left\langle\sigma_{n}\left(\mathcal{O}_{\alpha}\right)\right\rangle_{1}=\left\langle\sigma_{n-1}\left(\mathcal{O}_{\alpha}\right) \mathcal{O}_{\beta}\right\rangle_{0}\left\langle\mathcal{O}^{\beta}\right\rangle_{1} & +\left\langle\sigma_{n-2}\left(\mathcal{O}_{\alpha}\right) \mathcal{O}_{\gamma}\right\rangle_{0} \\
& \times\left(\left\langle\sigma_{1}\left(\mathcal{O}^{\gamma}\right)\right\rangle_{1}-\left\langle\mathcal{O}^{\gamma} \mathcal{O}_{\beta}\right\rangle_{0}\left\langle\mathcal{O}^{\beta}\right\rangle_{1}\right)
\end{aligned}
$$

Eq.(2.9) is our TRR at genus=1. It appears somewhat different from the standard TRR [4]

$$
\left\langle\sigma_{n}\left(\mathcal{O}_{\alpha}\right)\right\rangle_{1}=\frac{1}{24}\left\langle\sigma_{n-1}\left(\mathcal{O}_{\alpha}\right) \mathcal{O}_{\beta} \mathcal{O}^{\beta}\right\rangle_{0}+\left\langle\sigma_{n-1}\left(\mathcal{O}_{\alpha}\right) \mathcal{O}_{\beta}\right\rangle_{0}\left\langle\mathcal{O}^{\beta}\right\rangle_{1} .
$$

However, when one uses the structure of the genus=1 free energy

$$
F_{1}=\frac{1}{24} \log \operatorname{det}\left(u_{\alpha \beta}\right)+f_{1}\left(u_{\alpha}\right)
$$

one may easily check (2.9) and (2.10) are equivalent.

By repeating the same procedure as above we can derive the TRR at genus $=2$

$$
\begin{aligned}
& \left\langle\sigma_{n+5}\left(\mathcal{O}_{\alpha}\right)\right\rangle_{2}=\left\langle\sigma_{n+4}\left(\mathcal{O}_{\alpha}\right) \mathcal{O}_{\beta}\right\rangle_{0} A_{0}^{\beta}+\left\langle\sigma_{n+3}\left(\mathcal{O}_{\alpha}\right) \mathcal{O}_{\beta}\right\rangle_{0} A_{1}^{\beta} \\
& \quad+\left\langle\sigma_{n+2}\left(\mathcal{O}_{\alpha}\right) \mathcal{O}_{\beta}\right\rangle_{0} A_{2}^{\beta}+\left\langle\sigma_{n+1}\left(\mathcal{O}_{\alpha}\right) \mathcal{O}_{\beta}\right\rangle_{0} A_{3}^{\beta}+\left\langle\sigma_{n}\left(\mathcal{O}_{\alpha}\right) \mathcal{O}_{\beta}\right\rangle_{0} A_{4}^{\beta}
\end{aligned}
$$

where

$$
\begin{aligned}
A_{0}^{\beta} & \equiv\left\langle\mathcal{O}^{\beta}\right\rangle_{2} \\
A_{1}^{\beta} & \equiv\left\langle\sigma_{1}\left(\mathcal{O}^{\beta}\right)\right\rangle_{2}-\left\langle\mathcal{O}^{\beta} \mathcal{O}_{\gamma}\right\rangle_{0} A_{0}^{\gamma} \\
A_{2}^{\beta} & \equiv\left\langle\sigma_{2}\left(\mathcal{O}^{\beta}\right)\right\rangle_{2}-\left\langle\sigma_{1}\left(\mathcal{O}^{\beta}\right) \mathcal{O}_{\gamma}\right\rangle_{0} A_{0}^{\gamma}-\left\langle\mathcal{O}^{\beta} \mathcal{O}_{\gamma}\right\rangle_{0} \cdot A_{1}^{\gamma} \\
A_{3}^{\beta} & \equiv\left\langle\sigma_{3}\left(\mathcal{O}^{\beta}\right)\right\rangle_{2}-\left\langle\sigma_{2}\left(\mathcal{O}^{\beta}\right) \mathcal{O}_{\gamma}\right\rangle_{0} A_{0}^{\gamma}-\left\langle\sigma_{1}\left(\mathcal{O}^{\beta}\right) \mathcal{O}_{\gamma}\right\rangle_{0} \cdot A_{1}^{\gamma}-\left\langle\mathcal{O}^{\beta} \mathcal{O}_{\gamma}\right\rangle_{0} \cdot A_{2}^{\gamma} \\
A_{4}^{\beta} & \equiv\left\langle\sigma_{4}\left(\mathcal{O}^{\beta}\right)\right\rangle_{2}-\left\langle\sigma_{3}\left(\mathcal{O}^{\beta}\right) \mathcal{O}_{\gamma}\right\rangle_{0} A_{0}^{\gamma}-\left\langle\sigma_{2}\left(\mathcal{O}^{\beta}\right) \mathcal{O}_{\gamma}\right\rangle_{0} \cdot A_{1}^{\gamma}-\left\langle\sigma_{1}\left(\mathcal{O}^{\beta}\right) \mathcal{O}_{\gamma}\right\rangle_{0} \cdot A_{2}^{\gamma} \\
-\left\langle\mathcal{O}^{\beta} \mathcal{O}_{\gamma}\right\rangle_{0} \cdot A_{3}^{\gamma} . &
\end{aligned}
$$


Thus all the descendants $\left\{\sigma_{n}\left(\mathcal{O}_{\alpha}\right), n \geq 5\right\}$ may be eliminated in favor of $\left\{\sigma_{i}\left(\mathcal{O}_{\alpha}\right), i=1,2,3,4\right\}$ at genus $g=2$. An explicit form of the above TRR is presented in the Appendix.

Similarly, TRR's at arbitrary genus $(g \geq 1)$ are given by

$$
\begin{aligned}
& \left\langle\sigma_{n+3 g-1}\left(\mathcal{O}_{\alpha}\right)\right\rangle_{g}=\sum_{j=0}^{3 g-2}\left\langle\sigma_{n+3 g-2-j}\left(\mathcal{O}_{\alpha} \mathcal{O}_{\beta}\right)\right\rangle_{0} A_{j}^{\beta}, \\
& A_{0}^{\beta} \equiv\left\langle\mathcal{O}^{\beta}\right\rangle_{g}, \\
& A_{j}^{\beta}=\left\langle\sigma_{j}\left(\mathcal{O}^{\beta}\right)\right\rangle_{g}-\sum_{k=0}^{j-1}\left\langle\sigma_{k}\left(\mathcal{O}^{\beta}\right) \mathcal{O}_{\gamma}\right\rangle_{0} A_{j-k-1}^{\gamma} .
\end{aligned}
$$

Thus the descendant degrees are reduced to $n \leq 3 g-2$ at genus $g$. These TRR are not quite as efficient as the standard TRR at genus 0 and 1 which reduce the descendant degrees all the way to zero. As we shall see, however, they are powerful enough to determine instanton numbers of arbitrary genera when combined together with the Virasoro conditions.

\section{Virasoro Conditions and Higher Genus Curves in $C P^{2}$}

We now turn to the application of our TRR. In order to fix our discussions let us consider the case of $C P^{2}$ and determine the number of its genus $=2$ curves.

Let us first recall the Virasoro conditions for $C P^{2}[1]$

$$
L_{n} Z=0, \quad n \geq-1
$$

where

$$
\begin{gathered}
L_{-1}=\sum_{\alpha=0}^{2} \sum_{m=1}^{\infty} t_{m}^{\alpha} \partial_{m-1, \alpha} \\
+\frac{1}{2 \lambda^{2}} \sum_{\alpha=0}^{2} t^{\alpha} t_{\alpha} \\
L_{0}=\sum_{\alpha=0}^{2} \sum_{m=0}^{\infty}\left(b_{\alpha}+m\right) t_{m}^{\alpha} \partial_{m, \alpha}+3 \sum_{\alpha=0}^{1} \sum_{m=1}^{\infty} t_{m}^{\alpha} \partial_{m-1, \alpha+1} \\
+\frac{1}{2 \lambda^{2}} \sum_{\alpha=0}^{1} 3 t^{\alpha} t_{\alpha+1}-\frac{5}{16}
\end{gathered}
$$




$$
\begin{aligned}
L_{n}=\sum_{m=0}^{\infty} \sum_{\alpha=0}^{2} \sum_{j=0}^{2-\alpha} 3^{j} C_{\alpha}^{(j)}(m, n) t_{m}^{\alpha} \partial_{m+n-j, \alpha+j} & \\
+\frac{\lambda^{2}}{2} \sum_{\alpha=0}^{2} \sum_{j=0}^{2-\alpha} \sum_{m=0}^{n-j-1} 3^{j} D_{\alpha}^{(j)}(m, n) \partial_{m}^{\alpha} \partial_{n-m-j-1, \alpha+j} & \\
& +\frac{1}{2 \lambda^{2}} \sum_{\alpha=0}^{1-n} 3^{n+1} t^{\alpha} t_{\alpha+n+1}, \quad n \geq 1 .
\end{aligned}
$$

Here $b_{0}=-1 / 2, b_{1}=1 / 2, b_{2}=3 / 2$ and $b^{\alpha}=1-b_{\alpha}$ and the coefficient functions are defined by

$$
\begin{aligned}
C_{\alpha}^{(j)}(m, n) \equiv \frac{\Gamma\left(b_{\alpha}+m+n+1\right)}{\Gamma\left(b_{\alpha}+m\right)} \sum_{m \leq \ell_{1}<\ell_{2}<\cdots<\ell_{j} \leq m+n}\left(\prod_{i=1}^{j} \frac{1}{b_{\alpha}+\ell_{i}}\right), \\
D_{\alpha}^{(j)}(m, n) \equiv \frac{\Gamma\left(b^{\alpha}+m+1\right) \Gamma\left(b_{\alpha}+n-m\right)}{\Gamma\left(b^{\alpha}\right) \Gamma\left(b_{\alpha}\right)} \\
\times \sum_{-m-1 \leq \ell_{1}<\ell_{2}<\cdots<\ell_{j} \leq n-m-1}\left(\prod_{i=1}^{j} \frac{1}{b_{\alpha}+\ell_{i}}\right) .
\end{aligned}
$$

Conventional notations are $t^{0} \equiv t^{P}, t^{1} \equiv t^{Q}$ and $t^{2} \equiv t^{R}$.

A parameter $\lambda$ denotes the string coupling constant and the free energy has the genus expansion

$$
Z=\log F, \quad F=\sum_{g=0} \lambda^{2 g-2} F_{g} .
$$

In the small phase space $\left(t_{n}^{\alpha}=0, n>0\right.$ except $\left.t_{1}^{P}=-1\right)$ the genus-g free energy has a structure

$$
F_{g}=F_{g}^{c l}+\sum_{d=1} \frac{N_{d}^{g}}{(3 d+g-1) !}\left(t^{R}\right)^{3 d+g-1} e^{d t^{Q}}
$$

where $N_{d}^{g}$ denotes the number of degree $d$, genus $g$ irreducible curves passing through $3 d+g-1$ points in $C P^{2}$. The classical part of the free energy $F_{g}^{c l}$ is non-vanishing only at $g=0$ and $1, F_{0}^{c l}=t^{P}\left(t^{Q}\right)^{2} / 2+\left(t^{P}\right)^{2} t^{R} / 2, F_{1}^{c l}=$ $-t^{Q} / 8$.

In order to determine the instanton expansion (3.8) for $g=2$ free energy, we first have to determine the values of the "initial" descendants $\left\langle\sigma_{i}\left(\mathcal{O}_{\alpha}\right)\right\rangle_{2}(i=1,2,3,4)$. We consider the Virasoro conditions (3.1) for 
$n=1,2 \cdots, 12$ in the small phase space and substitute into these equations the known values of the correlation functions at genus 0,1 . We also use TRR at $g=2(2.12)$ to rewrite one-point functions of higher descendants in terms of those of initial descendants. Then the Virasoro conditions provide 12 linear relations for 12 unknowns $\left\langle\sigma_{i}\left(\mathcal{O}_{\alpha}\right)\right\rangle_{2}(\alpha=0,1,2, i=1,2,3,4)$ and one can determine them order by order in the instanton expansion.

If one further considers the next Virasoro condition $L_{n}$ with $n=13$ and substitutes into it the determined values of the initial descendants, it then gives a prediction on the $g=2$ free energy. One finds up to degree 10

$$
\begin{aligned}
F_{2}\left(t^{Q}, t^{R}\right)= & \frac{27}{13 !}\left(t^{R}\right)^{13} e^{4 t^{Q}}+\frac{36855}{16 !}\left(t^{R}\right)^{16} e^{5 t^{Q}}+\frac{58444767}{19 !}\left(t^{R}\right)^{19} e^{6 t^{Q}} \\
& +\frac{122824720116}{22 !}\left(t^{R}\right)^{22} e^{7 t^{Q}}+\frac{346860150644700}{25 !}\left(t^{R}\right)^{25} e^{8 t^{Q}} \\
& +\frac{1301798459308709880}{28 !}\left(t^{R}\right)^{28} e^{9 t^{Q}} \\
& +\frac{6383405726993645784000}{31 !}\left(t^{R}\right)^{31} e^{10 t^{Q}} .
\end{aligned}
$$

First 3 terms in the RHS of (3.9) agree with those of Caporaso and Harris [8] (Caporaso-Harris gives the number of curves which contains reducible components. A convenient method of subtraction of reducible part is described in [13]). The rest is our predictions. One can check higher Virasoro conditions $L_{n} n>13$ are satisfied simultaneously by (3.9).

\section{Discussions}

Precise agreement of our predictions for the genus 2 free energies of $C P^{2}$ with the known geometrical data gives a strong support for the validity of our Virasoro conditions and TRR's. It now seems that by making use of these equations one may be able to compute in principle the number of curves of any genus and degree in arbitrary Fano varieties. For instance, the genus-2 free energy of $C P^{3}$ up to degree 3 is computed as

$$
\begin{gathered}
F_{2}\left(t^{Q}, t^{R}, t^{S}\right)=-\frac{1}{288}+\frac{1}{360} \frac{\left(t^{S}\right)^{2}}{2 !} e^{t^{Q}}+\frac{1}{360} \frac{\left(t^{R}\right)^{2}\left(t^{S}\right)^{1}}{2 ! \cdot 1 !} e^{t^{Q}}+\frac{1}{180} \frac{\left(t^{R}\right)^{4}}{4 !} e^{t^{Q}} \\
+\frac{7}{240} \frac{\left(t^{R}\right)^{2}\left(t^{S}\right)^{3}}{2 ! \cdot 3 !} e^{2 t^{Q}}+\frac{7}{60} \frac{\left(t^{R}\right)^{4}\left(t^{S}\right)^{2}}{4 ! \cdot 2 !} e^{2 t^{Q}}+\frac{21}{40} \frac{\left(t^{R}\right)^{6}\left(t^{S}\right)^{1}}{6 ! \cdot 1 !} e^{2 t^{Q}}+\frac{161}{60} \frac{\left(t^{R}\right)^{8}}{8 !} e^{2 t^{Q}} \\
+\frac{1}{12} \frac{\left(t^{S}\right)^{6}}{6 !} e^{3 t^{Q}}+\frac{5}{12} \frac{\left(t^{R}\right)^{2}\left(t^{S}\right)^{5}}{2 ! \cdot 5 !} e^{3 t^{Q}}+\frac{5}{2} \frac{\left(t^{R}\right)^{4}\left(t^{S}\right)^{4}}{4 ! \cdot 4 !} e^{3 t^{Q}}+\frac{46}{3} \frac{\left(t^{R}\right)^{6}\left(t^{S}\right)^{3}}{6 ! \cdot 3 !} e^{3 t^{Q}} \\
+\frac{307}{3} \frac{\left(t^{R}\right)^{8}\left(t^{S}\right)^{2}}{8 ! \cdot 2 !} e^{3 t^{Q}}+747 \frac{\left(t^{R}\right)^{10}\left(t^{S}\right)^{1}}{10 ! \cdot 1 !} e^{3 t^{Q}}+5930 \frac{\left(t^{R}\right)^{12}}{12 !} e^{3 t^{Q}}
\end{gathered}
$$


Here the variables $t^{Q}, t^{R}, t^{S}$ are those dual to the Käler class $\omega$ and $\omega^{2}, \omega^{3}$ of $C P^{3}$, respectively. Number of genus $=3$ curves in $C P^{2}$ is currently under study:

Eq. (2.1) for the genus $g$ free energy is a deep result of the 2-dimensional gravity theory. One may imagine having a set of space-time fields $\left\{\phi^{\alpha}\right\}$ whose propagator is given by $u_{\alpha \beta}^{-1}$ and the j-point vertex by $u_{\alpha_{1} \alpha_{2} \cdots \alpha_{j}}$. It is known that the genus $g$ free energy $F_{g}$ equals the sum of the Feynman amplitudes of $g$-loop diagrams made of propagators and j-point vertices $(j=3,4, \cdots, 3 g-$ 1) $[10,11]$. The number of different vertices increases as the genus is increased and hence the system has the characteristic of non-polynomial closed string field theory. It is quite curious such a space-time interpretation exists behind our TRR.

Virasoro conditions $L_{n} Z=0(n \geq-1)$ are not independent in the large phase space since they form the algebra $\left[L_{n}, L_{m}\right]=(n-m) L_{n+m}$. In the small phase space, however, they become all independent and are used to determine unknown correlation functions. For the computation of genus 2 curves in $C P^{2}$ a large number (13) of Virasoro constraints were imposed. At the moment the logical relationship between TRR's and the infinite set of Virasoro conditions is not completely clear. In the case of 2-dimensional pure gravity Virasoro conditions alone completely determined the amplitude. Hence they imply TRR's. On the other hand in the case of 2-dimensional gravity coupled to minimal matter, additional $\mathrm{W}$-algebra conditions were necessary to completely determine the amplitudes. Thus the TRR's are independent of Virasoro conditions. The present case of topological string theories appears similar to that of the 2-dimensional gravity coupled to matter. It is an important issue if there exist analogues of $\mathrm{W}$ conditions in our present problem.

After completing this manuscript we received a new paper by Getzler [14] where his TRR at genus=2 is presented. It is easy to check that his equation (6) is consistent with our genus=2 TRR.

\section{Note Added}

We have obtained the genus $=3$ free energy of $C P^{2}$ up to degree 10 


$$
\begin{aligned}
F_{3}\left(t^{Q}, t^{R}\right) & =\frac{1}{14 !}\left(t^{R}\right)^{14} e^{4 t^{Q}}+\frac{7915}{17 !}\left(t^{R}\right)^{17} e^{5 t^{Q}}+\frac{34435125}{20 !}\left(t^{R}\right)^{20} e^{6 t^{Q}} \\
& +\frac{153796445095}{23 !}\left(t^{R}\right)^{23} e^{7 t^{Q}}+\frac{800457740515775}{26 !}\left(t^{R}\right)^{26} e^{8 t^{Q}} \\
& +\frac{5039930694167991360}{29 !}\left(t^{R}\right)^{29} e^{9 t^{Q}} \\
& +\frac{38747510483053595091600}{32 !}\left(t^{R}\right)^{32} e^{10 t^{Q}}
\end{aligned}
$$

First 3 terms of (4.2) agree with [8].

\section{Acknowledgments}

We would like to thank E. Getzler and S.K. Yang for discussions. T.E. also would like to thank I. Ciocan-Fontanine, C. Faber, S. Katz, Z. Qin and Y. Ruan for their interests in this work.

\section{Appendix: Genus 2 Topological Recursion Relation}

$$
\begin{gathered}
\left\langle\sigma_{n+5}\left(\mathcal{O}_{\alpha}\right)\right\rangle_{2}=\left\langle\sigma_{n}\left(\mathcal{O}_{\alpha}\right) \mathcal{O}^{\beta}\right\rangle_{0}\left\langle\sigma_{4}\left(\mathcal{O}_{\beta}\right)\right\rangle_{2} \\
+\left[\left\langle\sigma_{n+1}\left(\mathcal{O}_{\alpha}\right) \mathcal{O}^{\beta}\right\rangle_{0}-\left\langle\sigma_{n}\left(\mathcal{O}_{\alpha}\right) \mathcal{O}_{\gamma}\right\rangle_{0}\left\langle\mathcal{O}^{\gamma} \mathcal{O}^{\beta}\right\rangle_{0}\right]\left\langle\sigma_{3}\left(\mathcal{O}_{\beta}\right)\right\rangle_{2} \\
+\left[\left\langle\sigma_{n+2}\left(\mathcal{O}_{\alpha}\right) \mathcal{O}^{\beta}\right\rangle_{0}-\left\langle\sigma_{n+1}\left(\mathcal{O}_{\alpha}\right) \mathcal{O}_{\gamma}\right\rangle_{0}\left\langle\mathcal{O}^{\gamma} \mathcal{O}^{\beta}\right\rangle_{0}\right. \\
\left.+\left\langle\sigma_{n}\left(\mathcal{O}_{\alpha}\right) \mathcal{O}_{\mu}\right\rangle_{0}\left(\left\langle\mathcal{O}^{\mu} \mathcal{O}_{\rho}\right\rangle_{0}\left\langle\mathcal{O}^{\rho} \mathcal{O}^{\beta}\right\rangle_{0}-\left\langle\sigma_{1}\left(\mathcal{O}^{\mu}\right) \mathcal{O}^{\beta}\right\rangle_{0}\right)\right]\left\langle\sigma_{2}\left(\mathcal{O}_{\beta}\right)\right\rangle_{2} \\
+\left[\left\langle\sigma_{n+3}\left(\mathcal{O}_{\alpha}\right) \mathcal{O}^{\beta}\right\rangle_{0}-\left\langle\sigma_{n+2}\left(\mathcal{O}_{\alpha}\right) \mathcal{O}_{\gamma}\right\rangle_{0}\left\langle\mathcal{O}^{\gamma} \mathcal{O}^{\beta}\right\rangle_{0}\right. \\
+\left\langle\sigma_{n+1}\left(\mathcal{O}_{\alpha}\right) \mathcal{O}_{\mu}\right\rangle_{0}\left\{\left\langle\mathcal{O}^{\mu} \mathcal{O}_{\rho}\right\rangle_{0}\left\langle\mathcal{O}^{\rho} \mathcal{O}^{\beta}\right\rangle_{0}-\left\langle\sigma_{1}\left(\mathcal{O}^{\mu}\right) \mathcal{O}^{\beta}\right\rangle_{0}\right\} \\
+\left\langle\sigma_{n}\left(\mathcal{O}_{\alpha}\right) \mathcal{O}_{\mu}\right\rangle_{0}\left(-\left\langle\sigma_{2}\left(\mathcal{O}^{\mu}\right) \mathcal{O}^{\beta}\right\rangle_{0}+\left\langle\sigma_{1}\left(\mathcal{O}^{\mu}\right) \mathcal{O}_{\rho}\right\rangle_{0}\left\langle\mathcal{O}^{\rho} \mathcal{O}^{\beta}\right\rangle_{0}\right. \\
\left.\left.+\left\langle\mathcal{O}^{\mu} \mathcal{O}_{\rho}\right\rangle_{0}\left\langle\sigma_{1}\left(\mathcal{O}^{\rho}\right) \mathcal{O}^{\beta}\right\rangle_{0}-\left\langle\mathcal{O}^{\mu} \mathcal{O}_{\rho}\right\rangle_{0}\left\langle\mathcal{O}^{\rho} \mathcal{O}_{\sigma}\right\rangle_{0}\left\langle\mathcal{O}^{\sigma} \mathcal{O}^{\beta}\right\rangle_{0}\right)\right]\left\langle\sigma_{1}\left(\mathcal{O}_{\beta}\right)\right\rangle_{2}
\end{gathered}
$$




$$
\begin{gathered}
+\left[\left\langle\sigma_{n+4}\left(\mathcal{O}_{\alpha}\right) \mathcal{O}^{\beta}\right\rangle_{0}-\left\langle\sigma_{n+3}\left(\mathcal{O}_{\alpha}\right) \mathcal{O}_{\gamma}\right\rangle_{0}\left\langle\mathcal{O}^{\gamma} \mathcal{O}^{\beta}\right\rangle_{0}\right. \\
+\left\langle\sigma_{n+2}\left(\mathcal{O}_{\alpha}\right) \mathcal{O}_{\mu}\right\rangle_{0}\left(\left\langle\mathcal{O}^{\mu} \mathcal{O}_{\rho}\right\rangle_{0}\left\langle\mathcal{O}^{\rho} \mathcal{O}^{\beta}\right\rangle_{0}-\left\langle\sigma_{1}\left(\mathcal{O}^{\mu}\right) \mathcal{O}^{\beta}\right\rangle_{0}\right) \\
\cdot\left\langle\sigma_{n+1}\left(\mathcal{O}_{\alpha}\right) \mathcal{O}_{\mu}\right\rangle_{0}\left\{-\left\langle\sigma_{2}\left(\mathcal{O}^{\mu}\right) \mathcal{O}^{\beta}\right\rangle_{0}+\left\langle\sigma_{1}\left(\mathcal{O}^{\mu}\right) \mathcal{O}_{\rho}\right\rangle_{0}\left\langle\mathcal{O}^{\rho} \mathcal{O}^{\beta}\right\rangle_{0}\right. \\
\left.+\left\langle\mathcal{O}^{\mu} \mathcal{O}_{\rho}\right\rangle_{0}\left\langle\sigma_{1}\left(\mathcal{O}^{\rho}\right) \mathcal{O}^{\beta}\right\rangle_{0}-\left\langle\mathcal{O}^{\mu} \mathcal{O}_{\rho}\right\rangle_{0}\left\langle\mathcal{O}^{\rho} \mathcal{O}_{\sigma}\right\rangle_{0}\left\langle\mathcal{O}^{\sigma} \mathcal{O}^{\beta}\right\rangle_{0}\right\} \\
+\left\langle\sigma_{n}\left(\mathcal{O}_{\alpha}\right) \mathcal{O}_{\mu}\right\rangle_{0}\left(-\left\langle\sigma_{3}\left(\mathcal{O}^{\mu}\right) \mathcal{O}^{\beta}\right\rangle_{0}+\left\langle\sigma_{2}\left(\mathcal{O}^{\mu}\right) \mathcal{O}_{\rho}\right\rangle_{0}\left\langle\mathcal{O}^{\rho} \mathcal{O}^{\beta}\right\rangle_{0}\right. \\
+\left\langle\sigma_{1}\left(\mathcal{O}^{\mu}\right) \mathcal{O}_{\rho}\right\rangle_{0}\left\langle\sigma_{1}\left(\mathcal{O}^{\rho}\right) \mathcal{O}^{\beta}\right\rangle_{0}-\left\langle\sigma_{1}\left(\mathcal{O}^{\mu}\right) \mathcal{O}_{\gamma}\right\rangle_{0}\left\langle\mathcal{O}^{\gamma} \mathcal{O}_{\rho}\right\rangle_{0}\left\langle\mathcal{O}^{\rho} \mathcal{O}^{\beta}\right\rangle_{0} \\
+\left\langle\mathcal{O}^{\mu} \mathcal{O}_{\rho}\right\rangle_{0}\left\langle\sigma_{2}\left(\mathcal{O}^{\rho}\right) \mathcal{O}^{\beta}\right\rangle_{0}-\left\langle\mathcal{O}^{\mu} \mathcal{O}_{\gamma}\right\rangle_{0}\left\langle\sigma_{1}\left(\mathcal{O}^{\gamma}\right) \mathcal{O}_{\rho}\right\rangle_{0}\left\langle\mathcal{O}^{\rho} \mathcal{O}^{\beta}\right\rangle_{0} \\
\left.\left.-\left\langle\mathcal{O}^{\mu} \mathcal{O}_{\gamma}\right\rangle_{0}\left\langle\mathcal{O}^{\gamma} \mathcal{O}_{\rho}\right\rangle_{0}\left\langle\sigma_{1}\left(\mathcal{O}^{\rho}\right) \mathcal{O}^{\beta}\right\rangle_{0}+\left\langle\mathcal{O}^{\mu} \mathcal{O}_{\nu}\right\rangle_{0}\left\langle\mathcal{O}^{\nu} \mathcal{O}_{\rho}\right\rangle_{0}\left\langle\mathcal{O}^{\rho} \mathcal{O}_{\gamma}\right\rangle_{0}\left\langle\mathcal{O}^{\gamma} \mathcal{O}^{\beta}\right\rangle_{0}\right)\right]
\end{gathered}
$$

\section{References}

[1] T. Eguchi, K. Hori and C.-S. Xiong, Phys. Lett. B402 (1997) 71.

[2] T. Eguchi, M. Jinzenji and C.-S. Xiong, Nucl. Phys. B510 (1998) 608.

[3] S. Katz, unpublished, March 1997.

[4] E. Witten, Nucl. Phys. B340 (1990) 281.

[5] E. Getzler, talk given at Taniguchi Symposium "Integrable Systems and Algebraic Geometry", Kyoto, July 1997.

[6] I. Vainsencher, "Enumeration of n-fold Tangent Hyperplanes to a Surface", alg-geom/9312012.

[7] P. Di Francesco and C. Itzykson, "Quantum Intersection Rings", in "The Moduli Space of Curves", R. Dijkgraaf, C. Faber and G. van der Geer, eds., Birkhäuser, Boston-Basel-Berlin 1995.

[8] L. Caporaso and J. Harris, "Counting Plane Curves of Any Genus", alg-geom $/ 9608025$.

[9] R. Dijkgraaf and E. Witten, Nucl. Phys. B342 (1990) 486.

[10] C. Itzykson and J.-B. Zuber, Int. J. Mod. Phys. A7 (1992) 5661.

[11] T. Eguchi, Y. Yamada and S.-K. Yang, Rev. Math. Phys. 7 (1995) 279.

[12] T. Eguchi and S.-K. Yang, Mod. Phys. Lett. A9 (1994) 2893; T. Eguchi, K. Hori and S.-K. Yang, Int. J. Mod. Phys. A10 (1995) 4203. 
[13] E. Getzler, "Intersection Theory on $\overline{\mathcal{M}}_{1,4}$ and Elliptic Gromov-Witten Invariants", alg-geom/9612004, to appear in revised version in J. Amer. Math. Soc.

[14] E. Getzler, "Topological Recursion Relations in Genus 2", math.AG/9801003. 